\title{
Performance evaluation of MEMS accelerometers
}

\author{
A. Albarbar ${ }^{1 *}$, A. Badri', Jyoti K. Sinha ${ }^{1}$, A. Starr ${ }^{2}$ \\ ${ }^{1}$ School of Mechanical, Aerospace and Civil Engineering, \\ University of Manchester, P.O. Box 88, Manchester, M60 1QD \\ ${ }^{2}$ School of Automotive, Aerospace and Design Engineering, \\ University of Hertfordshire, Hatfield, Herts AL10 9AB
}

\begin{abstract}
Researchers have been looking for alternatives of expensive conventional accelerometers in vibration measurements. Micro-ElectroMechanical Systems (MEMS) accelerometer is one of the available options. Here the performance of one of these MEMS accelerometers compared with a well known commercial accelerometer.
\end{abstract}

\section{Key Words}

Condition Monitoring, Micro-elecromechanical System (MEMS) Accelerometer, Vibration Measurements, Frequency Response Function.

*Corresponding Author

a.albarbar@mmu.ac.uk

Office no. $+44(0) 1612476297$ 


\subsection{Introduction}

Condition based monitoring is now accepted practice for critical machines and structures for Industries to enhance availability, low maintenance costs and plant safety. Often, the decisions regarding the repair or replacement of a machine part, overhauls, and standard maintenance are made on the basis of the measured condition of the machine. One such monitoring technique is vibration based condition monitoring. Measuring vibration is very essential in detecting and diagnosing any deviation from normal conditions. The use of conventional piezoelectric accelerometers in vibration measurements is well known and accepted, but at high cost especially if simultaneous multiple data collection points are required; this is mainly because of their cost and the price of the associated electronic signal conditioning circuits.

The recent advances in embedded system technologies such as micro-electrical mechanical systems (MEMS) sensors hold a great promise for the future of vibration measurement based condition monitoring which is a much cheaper alternative. It has built-in signal conditioning unit as well. The cost of MEMS accelerometer may be just $10 \%$ or less compared to the commercially available cheapest conventional accelerometer together with the signal condition unit. There are number of research studies in the literature [1-8] about the MEMS accelerometers construction and the measurement principle.

The use of the MEMS accelerometers is still limited to testing stage in the laboratory experiments. Thanagasundram and Schlindwein [9] have used the MEMS accelerometer together with a conventional accelerometer for measuring the vibration of a pump during its normal operation. They [9] found the frequency content from both sensors were in agreement. However no rigorous investigation has been done to compare the performance

of this MEMS accelerometer which is required to measure the different kinds of signals sinusoidal, random, and impulsive signals [10]. Hence the performance of one of these 
MEMS accelerometers compared with a well known commercial accelerometer used for years together which are discussed here through a simple test facility.

\subsection{MEMS Accelerometer}

MEMS accelerometers are divided into two main types: peizoresistive and capacitive based accelerometers [8]. The conventional piezoelectric accelerometers generally consist of a single-degree of freedom system of a mass suspended by a spring. Here in piezoresistive MEMS Accelerometer also, it has a cantilever beam having a proof mass at the beam tip and a peizoresistive patch on the beam web. The schematic of a peizoresistive MEMS accelerometer is shown in Figure 1(a). The movement of the proof mass when subjected to vibration changes the resistance of the embedded piezoresistor. The electric signal generated from the piezoresistive patch due to change in resistance is proportional to the acceleration of the vibrating object. The capacitive based MEMS accelerometers measure changes of the capacitance between a proof mass and a fixed conductive electrode separated by a narrow gap [8]. The schematic of a capacitive MEMS accelerometer is shown in Figure 1(b). The papers [1-8] gave the details of the working principle and so not discussed here.

\subsection{Test Setup}

A schematic of the Test setup is shown in Figure 2. The setup consists of a small shaker (M/s GW make) together with a shaker power amplifier, signal generator and a PC based data acquisition for data collection and storage for further signal processing in MatLab. Two accelerometers (one PCB accelerometer and other capacitive type MEMS accelerometer) were attached back to back on the armature attached to the shaker as shown in Figure 2. The PCB accelerometer used for this experiment is an ICP (Integrated Circuit Piezoelectric) type with the technical specifications $-100 \mathrm{mV} / \mathrm{g}$, Linear Frequency range upto $2 \mathrm{kHz}, 50 \mathrm{~g}$ level. This type of Accelerometers has been calibrated as per the ISO/IEC 17025 standard and well accepted in practice because of their performance. A typical MEMS accelerometer of technical specifications $-250 \mathrm{mV} / \mathrm{g}$, Frequency range 10 $\mathrm{kHz}, 5 \mathrm{~g}$ level is used for comparison [11] which is relatively new technology for the accelerometer. 


\subsection{Testing and Results}

As it is well known that the accelerometers are used for measuring the periodic (sinusoidal, step-sine, multi-sine, etc.), random and impulsive signals [10], hence these tests were carried out on the Test setup and results were compared.

\subsection{Periodic Excitation}

Sinusoidal signals were given to the shaker at two frequencies $-66 \mathrm{~Hz}$ and $157 \mathrm{~Hz}$ deliberately away from the line frequency of $50 \mathrm{~Hz}$ and its harmonics. Number of experiments was performed at these two frequencies with different amplitude levels of shaker excitation and simultaneously responses were measured from both accelerometers. Few typical measured responses both in time and frequency domain is shown Figures 3 to 5. No distortion is seen in the measured responses by the MEMS accelerometer as well. However there is significant shift in phase and the sensitivity compared to the reference accelerometer. In fact the estimated sensitivity based on reference accelerometer seems to be varying from $38 \mathrm{mV} / \mathrm{g}$ to $69 \mathrm{mV} / \mathrm{g}$ and phase shift is also not constant with respect to the reference accelerometer responses which are clearing seen in the time response plots in Figures 3, 4 and 5.

\subsection{Random Excitation}

Similar to the sinusoidal tests; the shaker was excited with random excitation in a frequency band from $10 \mathrm{~Hz}$ to $1.5 \mathrm{kHz}$ with different amplitudes. Typical responses of both accelerometers in time and frequency domains are shown in Figure 6. Both accelerometers responses look to be identical in time and frequency domains, but here again the estimated sensitivity found to be $225 \mathrm{mV} / \mathrm{g}$ though it is close to the design value of the sensitivity for this MEMS accelerometer but much different than the estimated sensitivity during the sinusoidal tests. 
To determine the linearity in the measurement over the frequency band of excitation and phase shift, the frequency response function (FRF- the transfer function in frequency domain) has also been calculated assuming the responses of the MEMS accelerometer as the output and the reference accelerometer responses as the input. Both the amplitude and phase FRF plots are shown in Figure 7. Although the response spectra shown in Figure 10 looks to be identical but the amplitude deviation between two accelerometers is found to be approximately upto $-20 \mathrm{~dB}$ at some frequencies and the phase shift of 180 degree upto around $100 \mathrm{~Hz}$ and then the shift is approximately linear from 180 degree at $100 \mathrm{~Hz}$ to zero degree at $1.5 \mathrm{kHz}$.

\subsection{Impulsive Excitation}

In the same experimental setup, the impact excitation was given at the centre of the armature using a soft tip hammer within the frequency band of excitation up to 400$500 \mathrm{~Hz}$. Typical time domain responses of both accelerometers are shown in Figure 8. The measured responses are typically decay type responses as expected for the impact excitation by both accelerometers with maximum amplitude level of $0.6 \mathrm{~g}$. However the estimated sensitivity once again found to be $155.9 \mathrm{mV} / \mathrm{g}$ which is different than earlier estimated values and the fast decay in the response seen in the MEMS accelerometer compared to the reference accelerometer. To understand this typical behaviour of the MEMS accelerometer, the averaged spectra of the 3 decay responses were computed for both accelerometers and compared which is shown in Figure 8. The presence of the frequency peaks is consistent in both responses, however the peaks amplitudes are much different. Since the MEMS accelerometer introduces the phase shift known from previous tests, so these phase shift at different frequencies might be resulted into the fast decay in the measured responses compared to the reference accelerometer.

\subsection{Further Analysis}

In vibration analysis, the ordinary coherence between two vibration signals is defined as [12]:

$\operatorname{Coh}\left(\gamma^{2}\right)=\frac{\left|S_{x y}(\omega)\right|^{2}}{S_{y y}(\omega) S_{x x}(\omega)^{\prime}}$ 
where $S_{x x}(\omega)$ and $S_{y y}(\omega)$ are the power spectral densities of two signals, $x(t)$ and $y(\mathrm{t})$, and $S_{x y}(\omega)$ is their cross-power spectrum at an angular frequency $(\omega)$. The coherence between two signals indicates the degree to which two signals are linearly correlated at a given frequency. The Coherence (Coh) is close to unity means the signal $x(t)$ is linearly correlated to the signal $\mathrm{y}(\mathrm{t})$. Reduction in the coherence from 1 indicates that the two signals are either noisy or having nonlinear relation. Hence the coherence was computed between two responses measured by the MEMS and the reference Accelerometers. Figure 9 shows the coherence plots for the random and impact tests. Generally coherences are above 0.8 at many frequencies indicating good relation between two signals, but also low at several other frequencies. Hence this indicates the measurements by the MEMS accelerometer used in the present study deviate compared to the reference accelerometer.

\subsection{Comments}

The performance tests of a typical capacitive type MEMS accelerometer are carried out for different excitations - sinusoidal, random and impulse. The measured responses of the MEMS accelerometer were compared with a well accepted ICP type accelerometer. All tests were conducted well within the technical specifications of both accelerometers. The MEMS accelerometer is seems to be performing well for the sinusoidal and random measurements though shift in phase is observed, and the frequency peaks content is also found to be same in comparison with the conventional accelerometer for the impact excitation. However the tests performed here clearly show lots of improvement needed before its use in practice. It is being planed to carry out more investigation with several numbers of the MEMS accelerometers to understand the future direction for improvements.

\section{References}

1. Plaza J., Collado A., Cabruja E., Esteve J., Piezoresistive accelerometers for MCM package, Journal of Microelectromech. Systems, vol. 11, no. 6, pp. 794-801, Dec. 2002. 
2. Xie H., Fedder G., "CMOS z-axis capacitive accelerometer with comb-finger sensing, Proc. IEEE Micro Electro Mechanical Systems (MEMS), 2000, pp. 496-501.

3. Biefeld V., A. Buhrdorf, and J. Binder, "Laterally driven accelerometer fabricated in single crystalline silicon, Sensors \& Actuators A: Phys., vol. 82, no. 1, pp. 149-154, May 2000.

4. Yazdi N., F. Ayazi, and K. Najafi, Micromachined inertial sensors, Proc. IEEE, vol. 86, no. 8, pp. 1640-1659, August 1998.

5. Liu C., and T.W. Kenny, A high-precision, wide-bandwidth micromachined tunneling accelerometer, Journal of Microelectromech. Systems, vol. 10, no. 3, pp. 425-433, 2001.

6. Li L., Y. Xu, Y. Zhao, C. Liang, T. Wei, and Y. Yang, "Micromachined accelerometer based on electron tunneling, Proc. SPI-International. Society Optical Engineering., vol. 3891, pp. 121-125, 1999.

7. Seshia A., Palaniapan M., Roessig T, Howe R., Gooch R., Schimert T., Montague S., A vacuum packaged surface micromachined resonant accelerometer, Journal of Microelectromech. Systems, vol. 11, no. 6, pp. 784-793, 2002.

8. Gao R., Zhang L., Micromachined microsensors for manufacturing, IEEE Instrumentation \& Measurement Magazine, 2004, 1094-6969.

9. Thanagasundram S., Schlindwein F., Comparison of integrated micro-electricalmechanical system and piezoelectric accelerometers for machine condition monitoring, IMechE Journal of Mechanical Engineering Science Part C, 220 11351146, 2006.

10. Sinha, Jyoti K., On Standardisation of calibration procedure for accelerometer. Journal of Sound and Vibration, 2005, 286(2005)417-427.

11. Albarbar A. Starr A., Pietruszkiewicz R., To Wards the implementation of integrated multimeasurand wireless monitoring system. Proc. Second world congress on Engineering and Asset Management, pp. 96-105, June 2007.

12. Suryam B.C.B.N, Meher K.K., Sinha J. K., Rao A., Coherence measurement for early contact detection between two components, Journal of Sound and Vibration, 290(2006)519-523. 


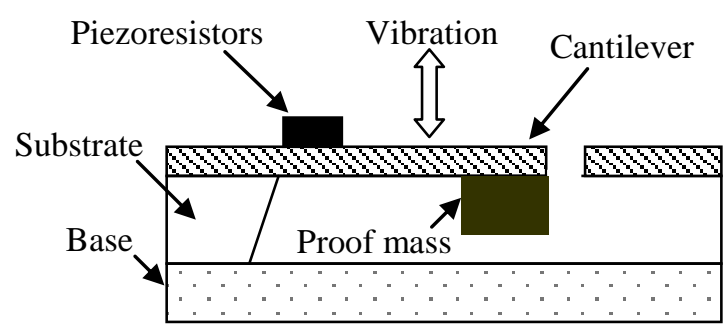

(a)

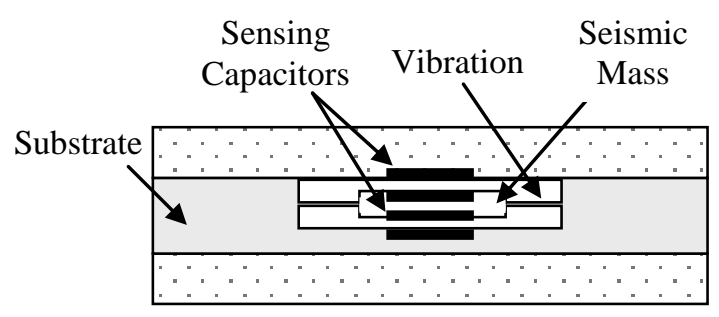

(b)

Figure 1 A typical MEMS accelerometer construction; (a) peizoresistive using cantilever design, (b) capacitive based on membrane design [8]

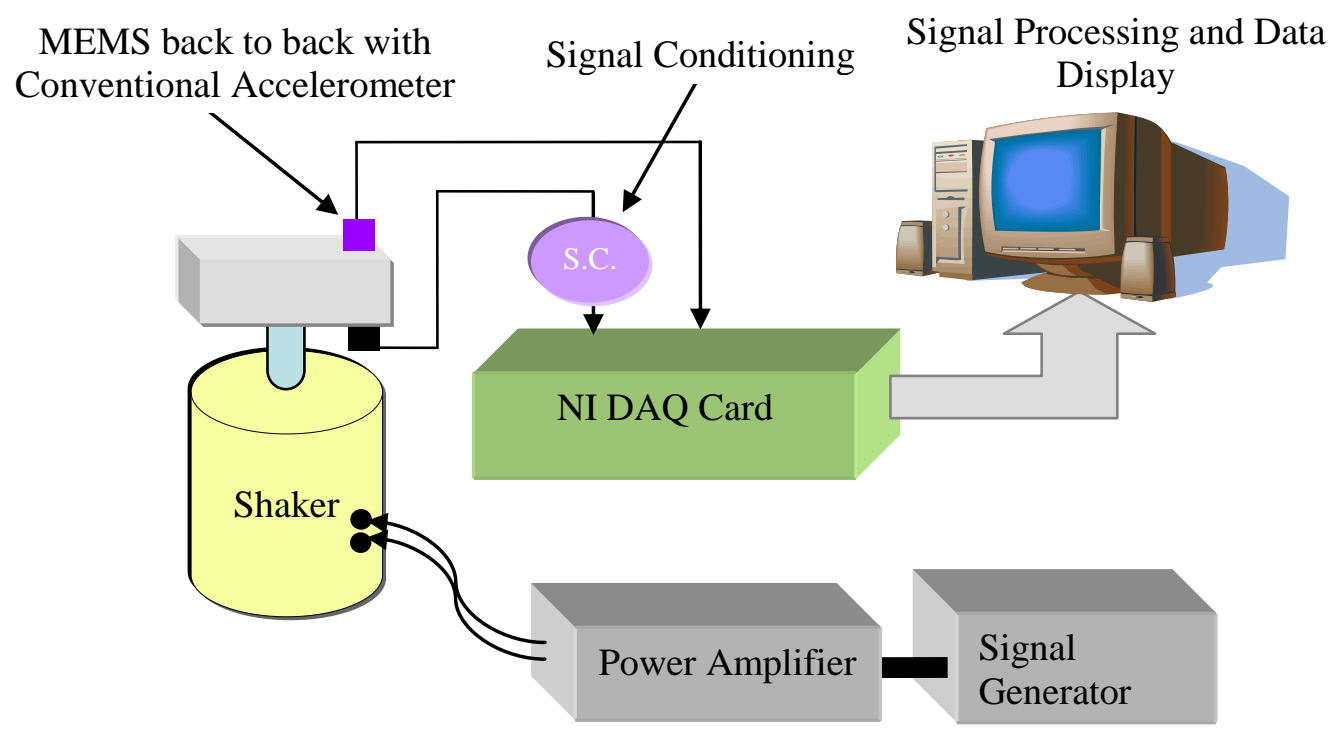

Figure 2 Test setup 

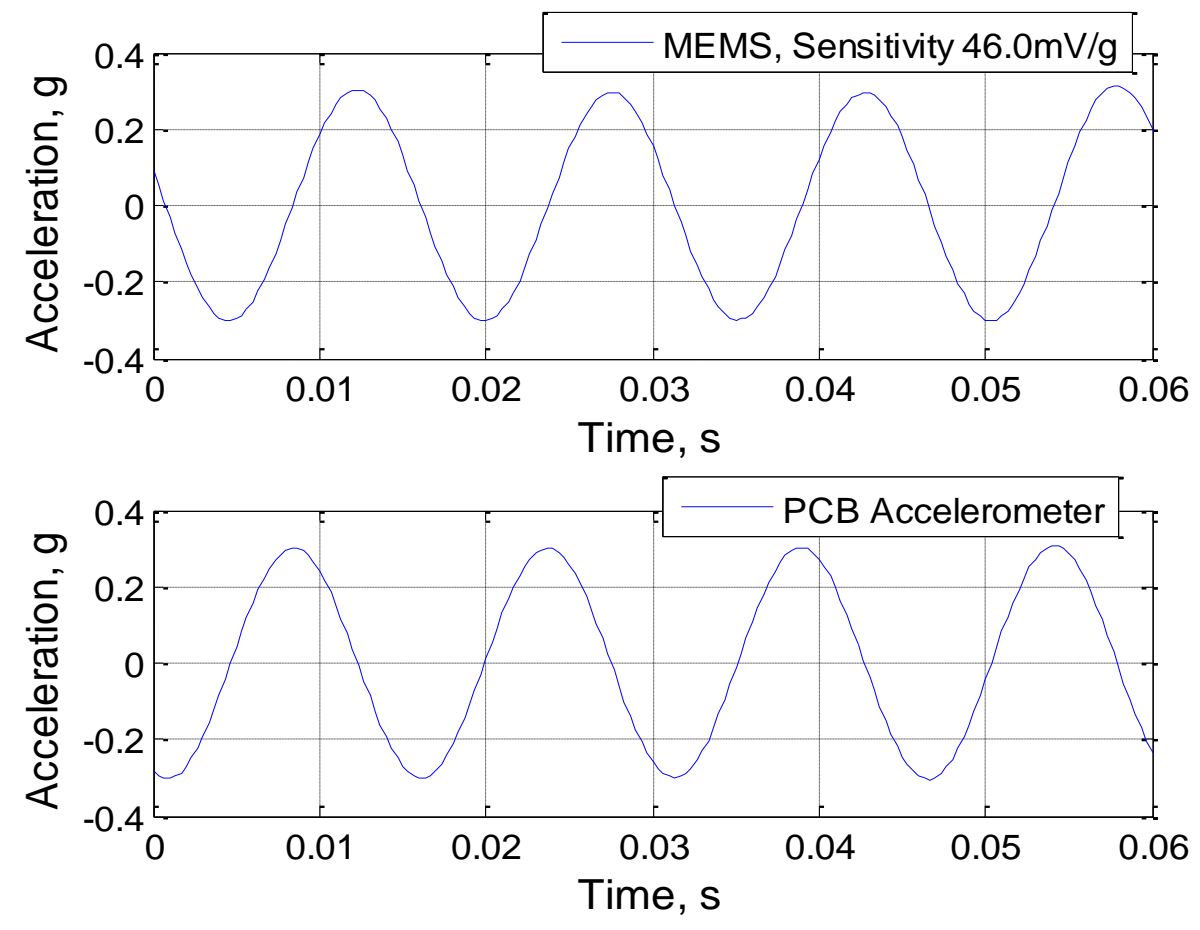

(a) Time domain signals
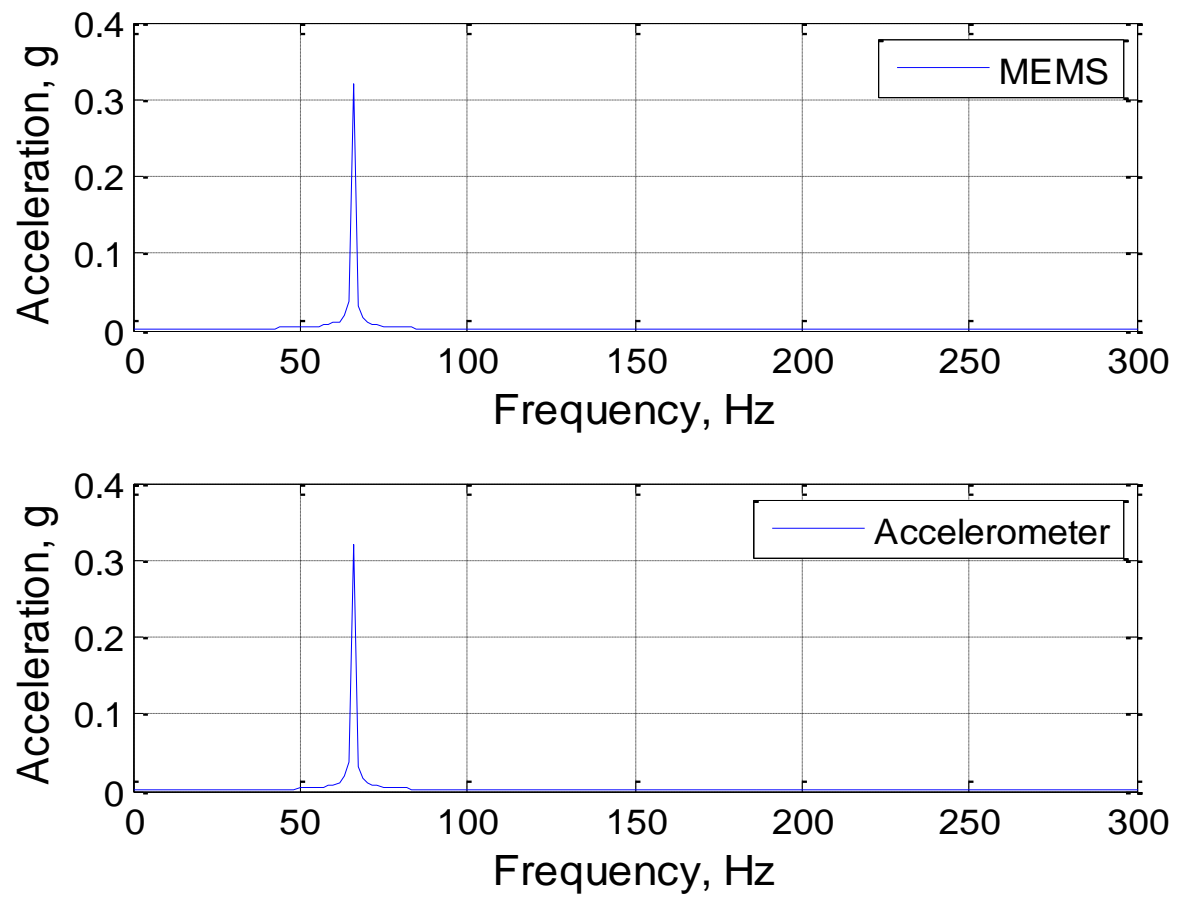

(b) Spectra

Figure 3 Measured acceleration responses by the MEMS accelerometer and the reference (PCB) accelerometer at $66 \mathrm{~Hz}$ for the excitation level $0.3 \mathrm{~g}$ 

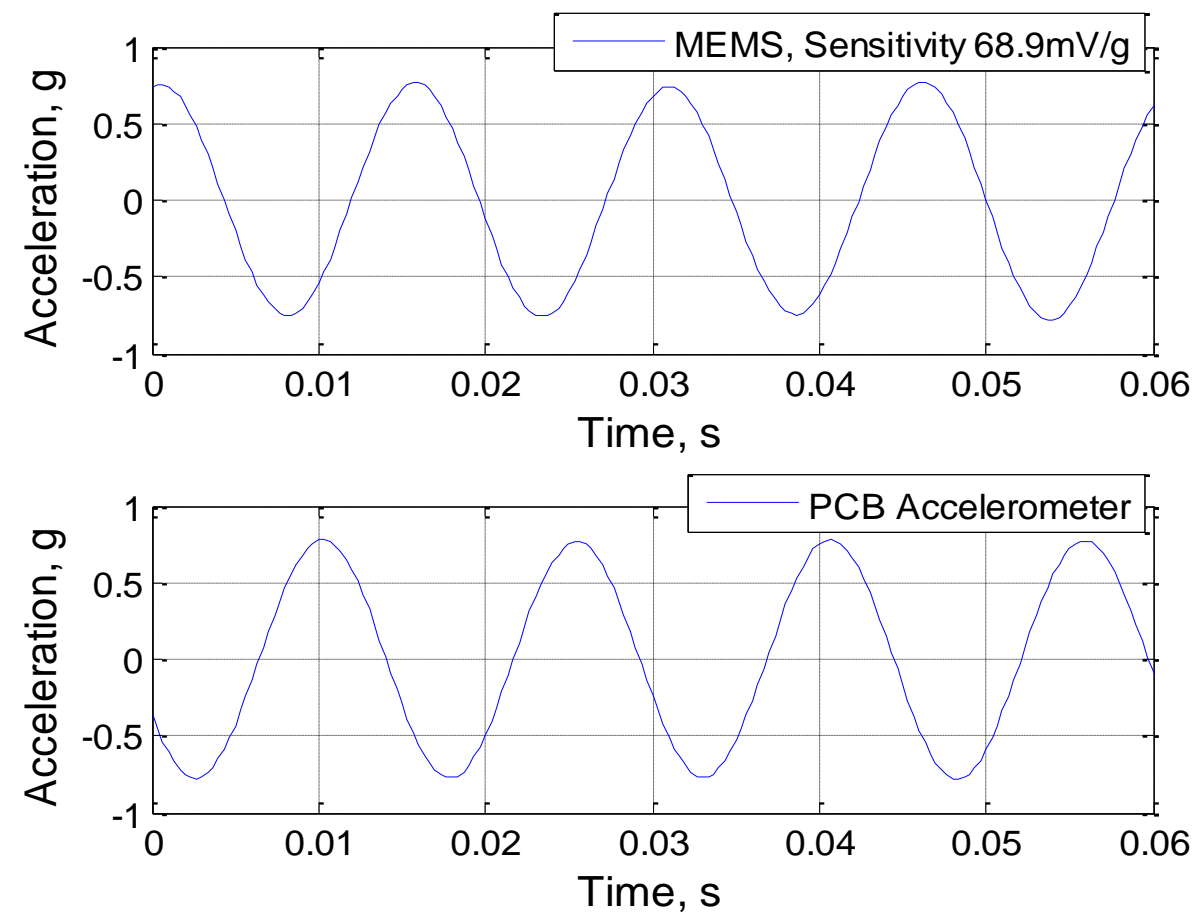

(a) Time domain signals
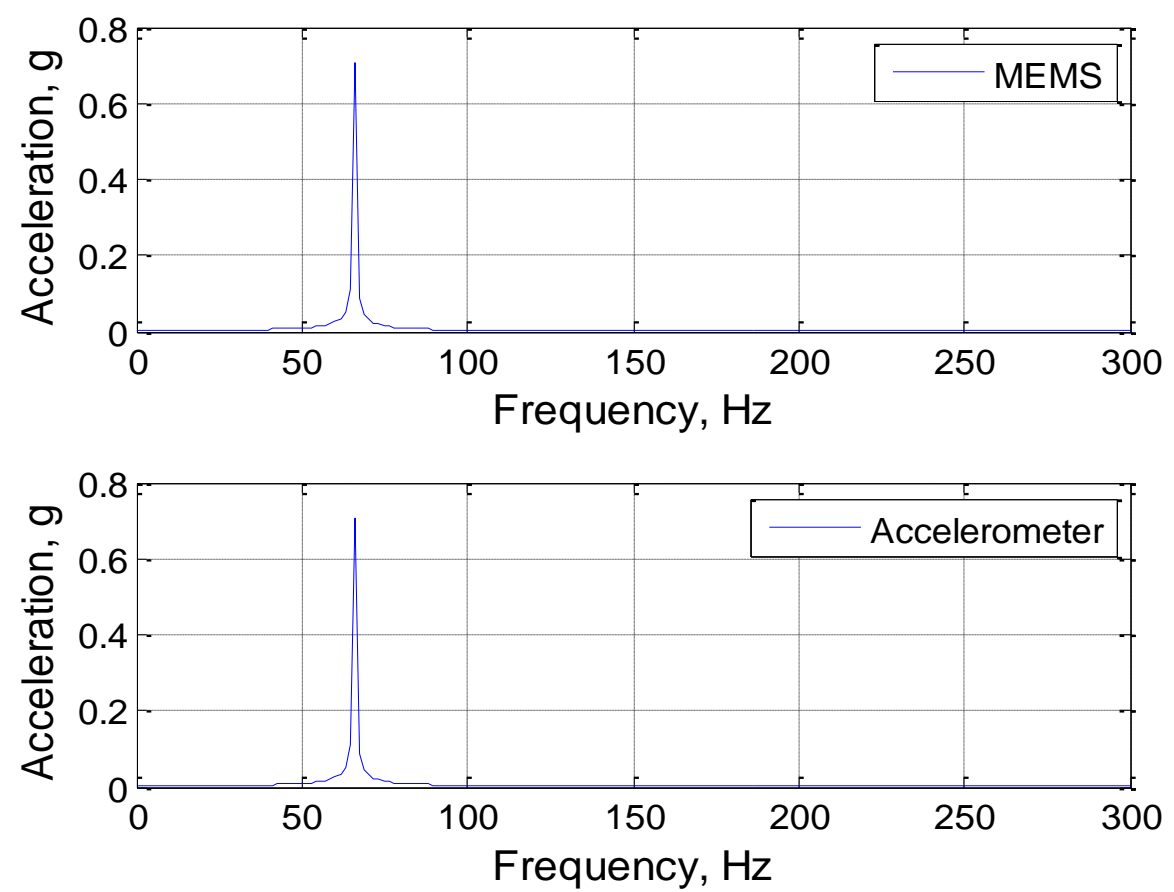

(b) Spectra

Figure 4 Measured acceleration responses by the MEMS accelerometer and the reference (PCB) accelerometer at $66 \mathrm{~Hz}$ for the excitation level $0.75 \mathrm{~g}$ 

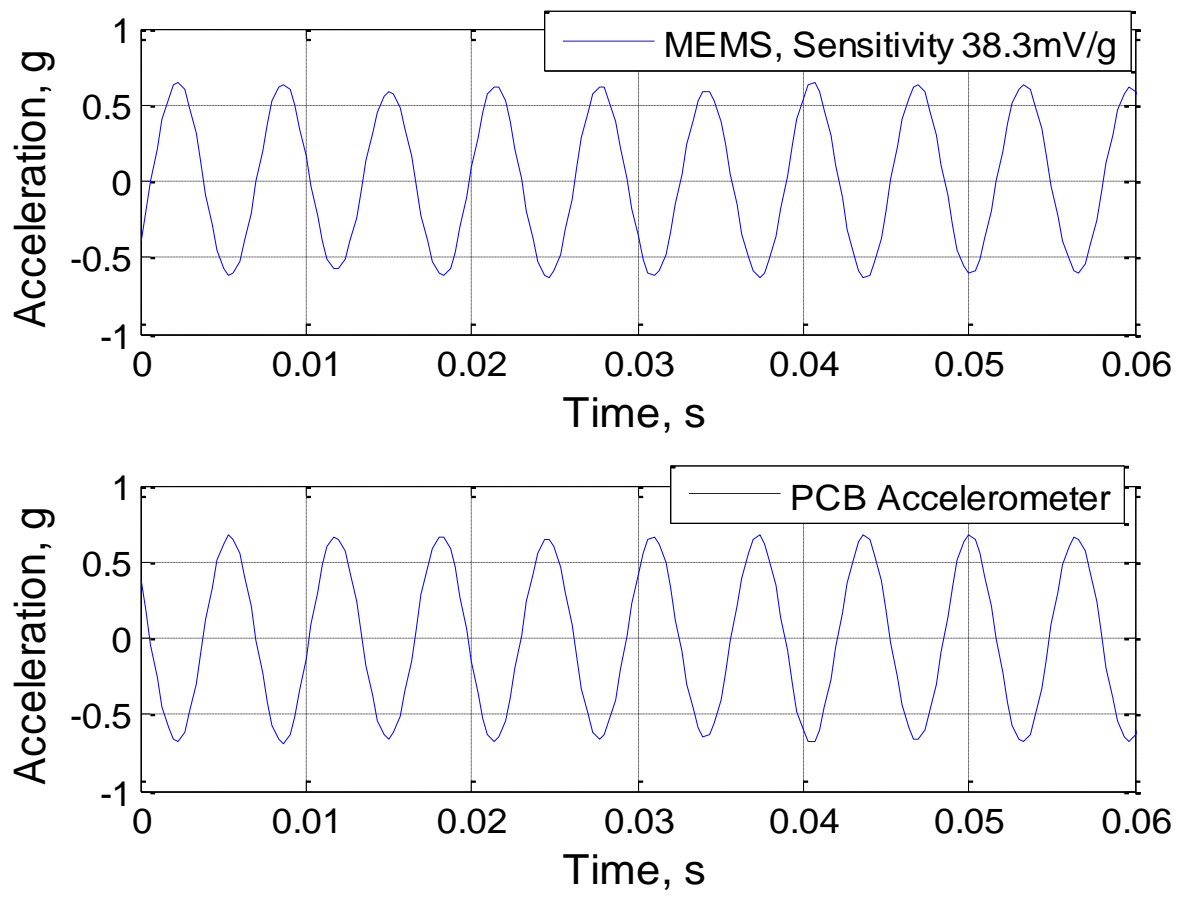

(a) Time domain signals
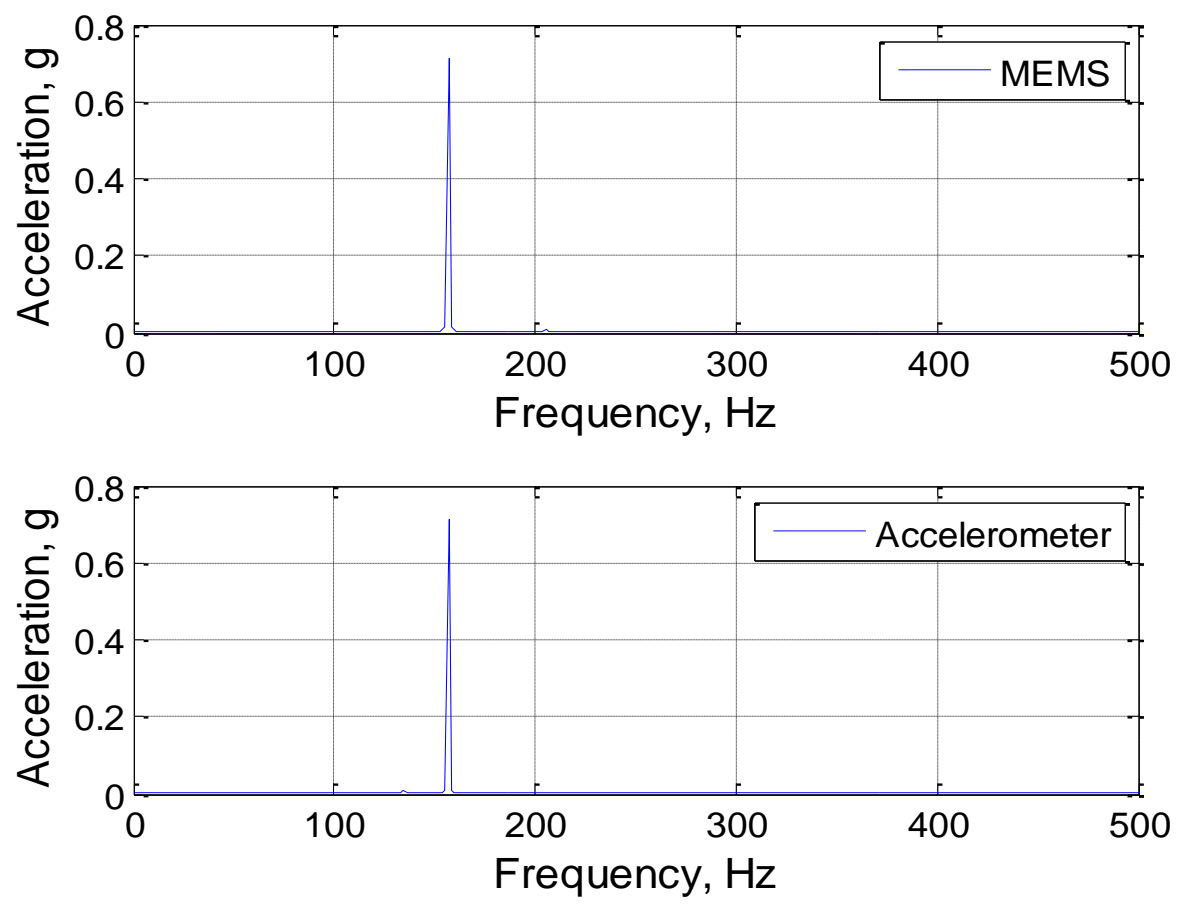

(b) Spectra

Figure 5 Measured acceleration responses by the MEMS accelerometer and the reference (PCB) accelerometer at $157 \mathrm{~Hz}$ for the excitation level $0.65 \mathrm{~g}$ 

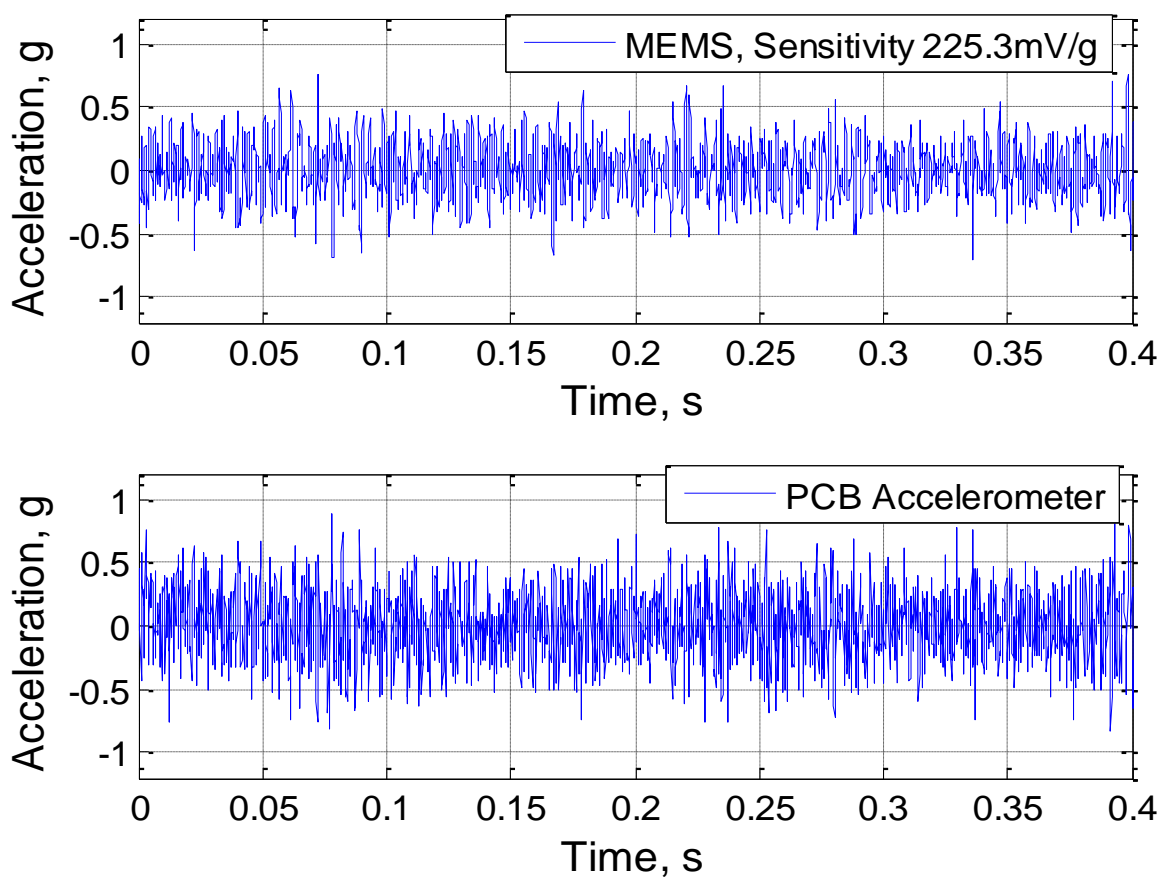

(a) Time domain signals
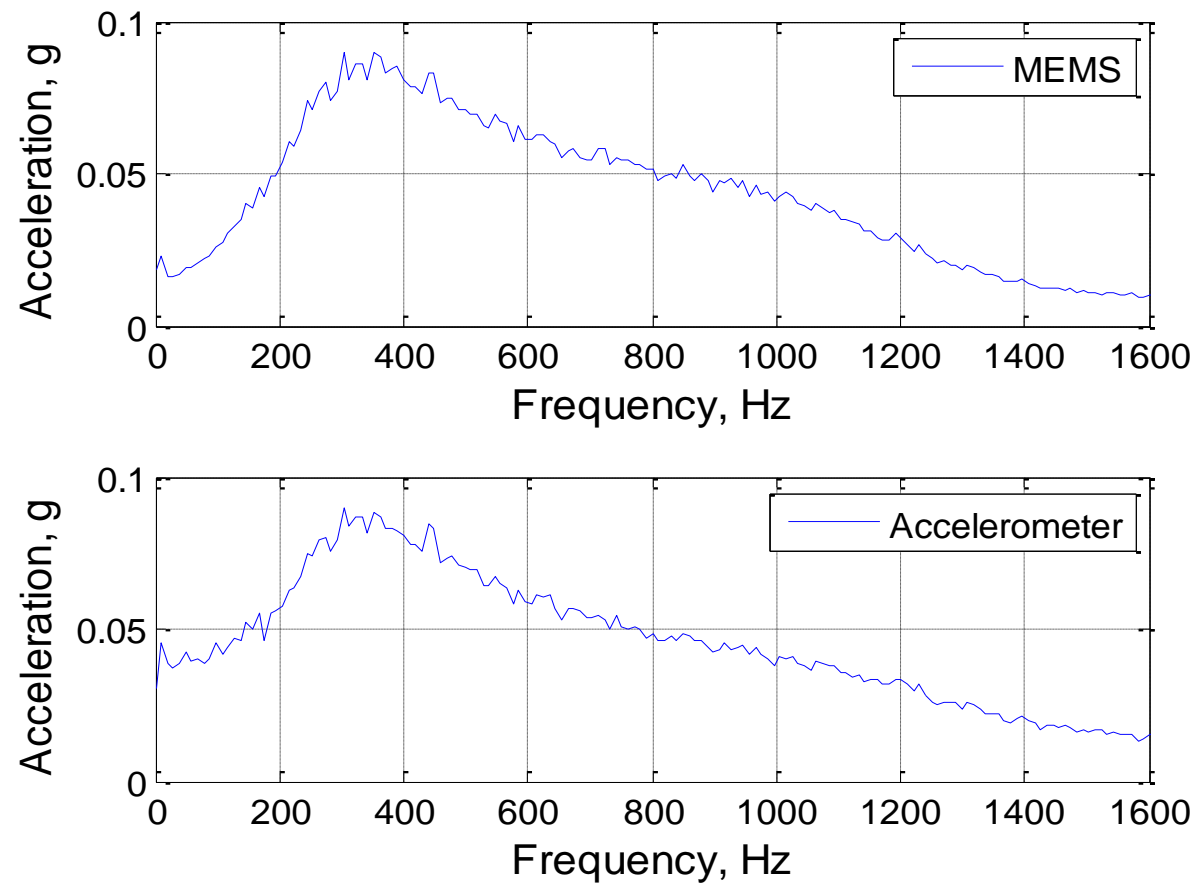

(b) Spectra

Figure 6 Measured acceleration responses by the MEMS accelerometer and the reference (PCB) accelerometer for the random excitation 

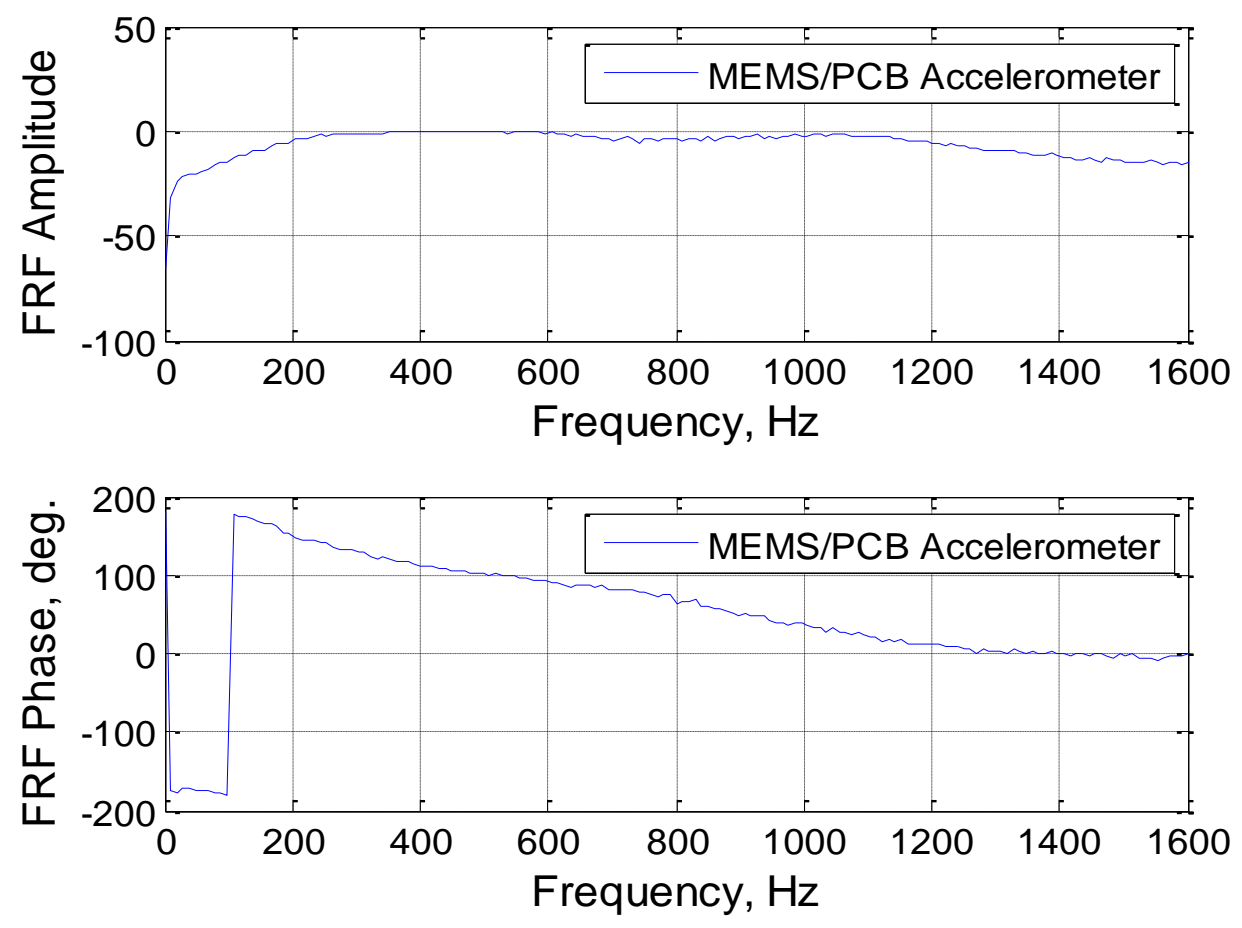

Figure 7 A typical measured FRF for the random excitation 

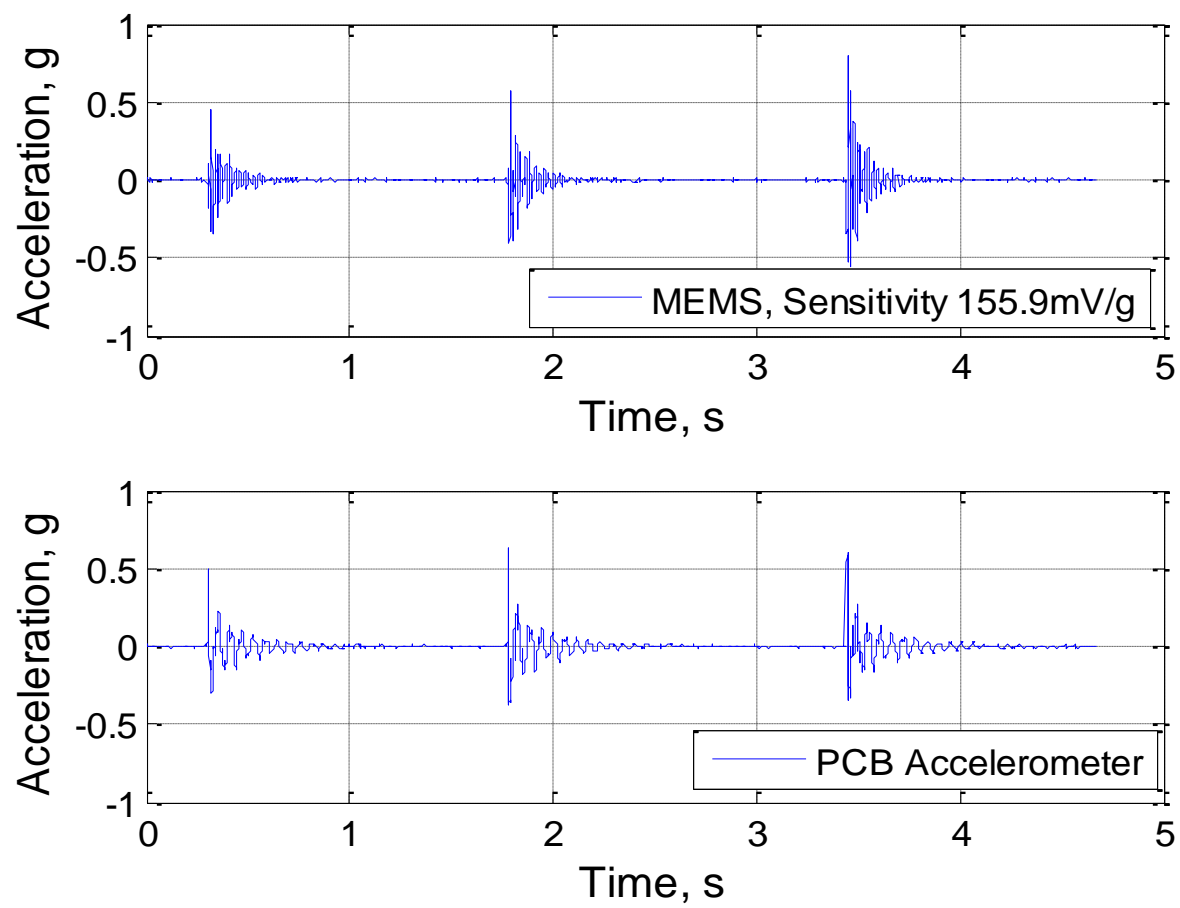

(a) Time domain signals
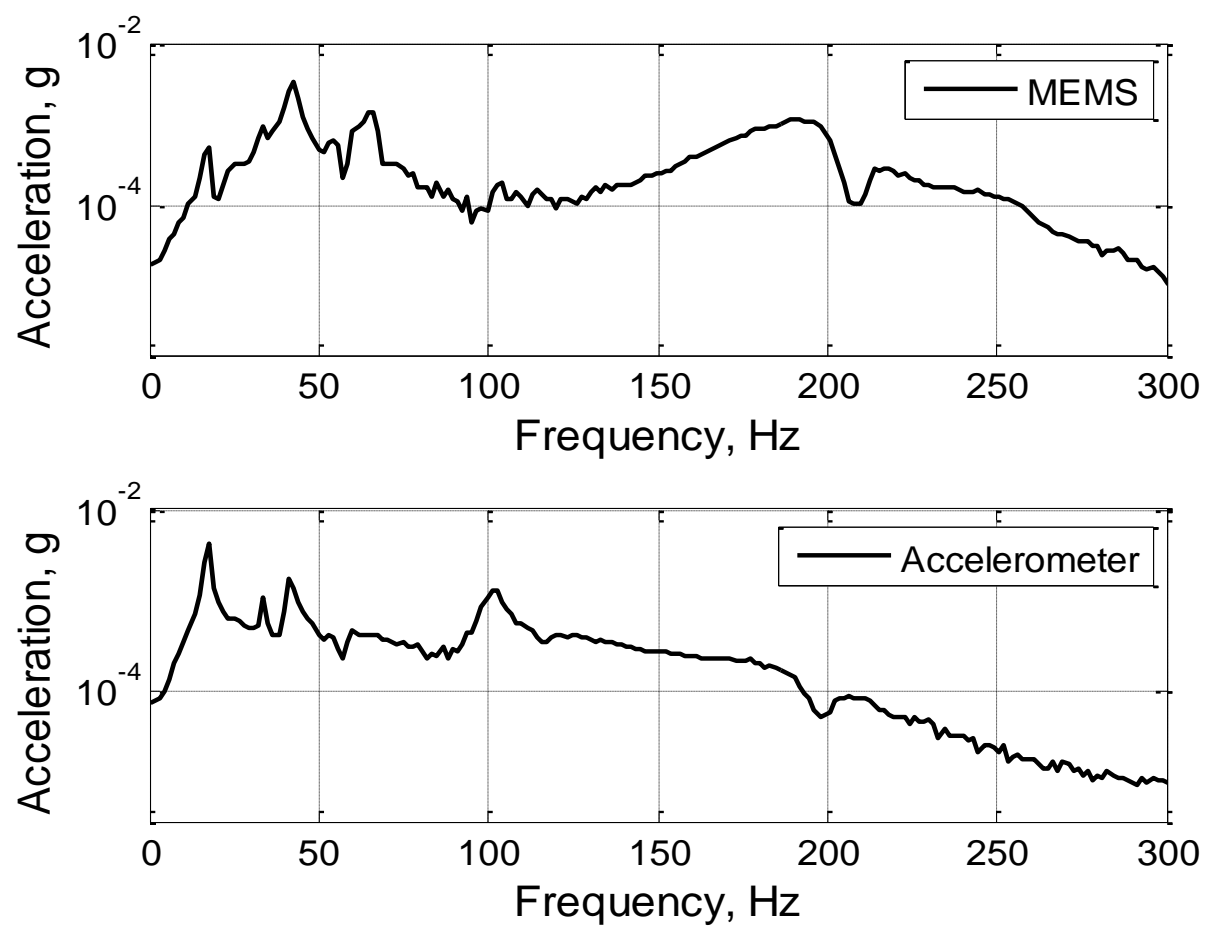

(b) Spectra

Figure 8 A comparison of measured responses by MEMS accelerometer and the PCB accelerometer when the shaker armature excited by impacts from a hammer 

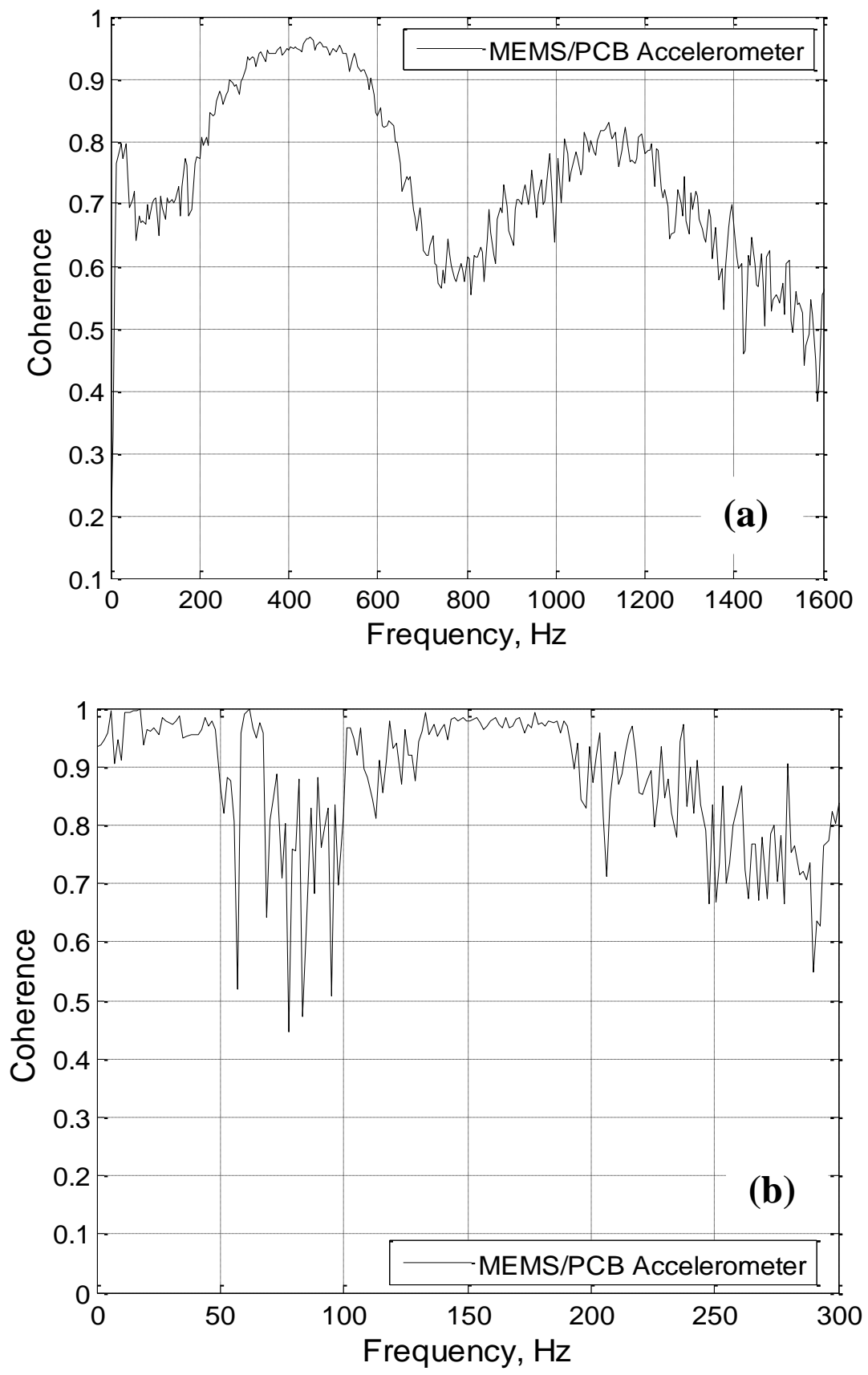

Figure 9 Coherence plots between the measured responses by the MEMS and the reference Accelerometers (a) Random Test, (b) Impact Test 